\title{
Cuando los momentos de ocio se convierten en la fuente de grandes ideas y oportunidades. Una aproximación crítica que desmitifica al gamer
}

\author{
When leisure moments becomes the source \\ of great ideas and opportunities. A critical \\ approach that demystifies the gamer
}

DOI: https://doi.org/10.17981/econcuc.42.2.2021.Org.1

Artículo de revisión.

Fecha de recepción: 14/10/2020

Fecha de devolución: 17/02/2021

Fecha de aceptación: 01/03/2021

Fecha de publicación: 10/03/2021

\section{Ramiro A. Buitrago Acuña \\ Alcaldía Bolivariana del Municipio Miranda - Edo. Zulia \\ Maracaibo, Zulia (Venezuela) dr.buitrago86@gmail.com}

\section{Josué N. Vera Rodríguez}

Consultant and Partnership Alliance

For Excellence, Inc / Pontificia

Universidad Católica de Puerto Rico

Ponce, Puerto Rico (Puerto Rico)

jverarodriguez@pucpr.edu

\section{Para citar este artículo:}

Buitrago, R. \& Vera, J. (2021). Cuando los momentos de ocio se convierten en la fuente de grandes ideas y oportunidades. Una aproximación crítica que desmitifica al gamer. Económicas CUC, 42(2), 9-32. DOI: https://doi. org/10.17981/econcuc.42.2.2021.Org.1

\section{Resumen}

El manuscrito en cuestión, reviste entre sus propósitos elevar argumentos que, desde una perspectiva sofista, procuren develar los mitos que por décadas han prejuiciado al fenómeno gamer, ante las miradas de quienes hacen ciencia. Se trata de un escrito tipo documental con diseño bibliográfico, donde se aplicó el método bibliográfico con enfoque interpretativo y la técnica de investigación documental, fue aprovechada en la revisión de los documentos seleccionados para tal estudio. A partir de ello, se reflexionó sobre los momentos de ocio, considerado por los detractores del estudio del ser, como pérdida de tiempo y qué en opinión de los autores de este extenso, configuran una serie de oportunidades, donde el gamer encuentra espacios para generar nuevas propuestas estratégicas, con las que pudiera favorecer el desarrollo de procesos blandos. Finalmente, desde una perspectiva analítica y documental, se reconoce al ocio como necesidad humana hacia la evolución, comprendiendo que la forma más elevada del pensamiento, es aquella donde las grandes ideas se erigen como galeones en las aguas de la epoché.

Palabras clave: Estrategias; TIC; cultura corporativa; responsabilidad social corporativa; creatividad; ocio tecnológico; videojuegos

\begin{abstract}
Among its purposes, the manuscript in question has to raise arguments that, from a sophist perspective, seek to unveil the myths that for decades have prejudiced the gamer phenomenon, before the eyes of those who do science. It is a documentary type writing with a bibliographic design, where the bibliographic method with an interpretive approach was applied and the documentary research technique was used in the review of the documents selected for such study. From this, it was reflected on leisure moments, considered by detractors of the study of being, as a waste of time and what, in the opinion of the authors of this extensive, configure a series of opportunities, where the gamer finds spaces to generate new strategic proposals, with which it could favor the development of soft processes. Finally, from an analytical and documentary perspective, leisure is recognized as a human need towards evolution, understanding that the highest form of thought is one where great ideas are erected like galleons in the waters of the epoché Keywords: Strategies; ICT; corporate culture; corporate social
\end{abstract} responsibility; creativity; technological leisure; videogame 


\section{INTRODUCCIÓN}

Los laudos que reposan en el argumento del manuscrito, están orientados hacia la comprensión, del significado que en la contemporaneidad ha adquirido el fenómeno gamer, como arquetipo del comportamiento humano, consecuente al impacto de las tecnologías de la información y comunicación, a las que se hará referencia como TIC, en la continuación del discurso; y que ante la necesidad de informatizar los procesos más neurálgicos, al interior de las organizaciones contemporáneas, han ejercido hasta entonces un rol de interés, en el desarrollo de estrategias, planes, políticas, programas de interés humano, a favor del capital intelectual en el mercado laboral del área Sur de Puerto Rico.

En ese sentido, corresponde exponer que el fenómeno gamer, más allá de presentar y personificar una forma de comportamiento humano, es el reflejo de la realidad informatizada, que implica para la cultura occidental el auge de las TIC y el internet, pues estos últimos revisten el contexto en donde bien o mal, desarrollan su personalidad los hombres en la contemporaneidad. En ello, se erigen los diferentes elementos que le circundan, los cuales a su vez, influyen en procesos blandos del contexto organizacional, donde el gamer asume y ejerce su rol social, cultural, profesional e incluso humano, donde a los efectos de este manuscrito destaca el ocio.

En ello, se erigen diferentes interrogantes: — ¿En que consiste el ocio entendido como escenario fructífero y no como holgazanería?; - ¿qué relación embarga con fenómenos tan dinámicos como el gamer?; ¿de dónde surge la idea en la que el ocio pudiera favorecer los procesos cognoscitivos?; - ¿qué representa el ocio para las ciencias administrativas y de que forma resulta edificante? Para poder atender estas y otras tantas preguntas que pudieran formularse, es necesario comprender al ocio en su origen como acervo científico.

Igualmente, el interés inicial de los autores de este escrito, consiste en auscultar desde un enfoque sofista el contenido del ocio, como fenómeno y proceso, a su vez describirle quizás como el paisaje al fondo del gamer, algún episodio de la realidad contemporánea por la que el hombre transita, teniendo que lidiar con el conglomerado de factores favorecedores o detractores, que implica el auge de la informatización en la cotidianidad, amén de que la vida resulte cada día mas sencilla, pudiendo afrontar los retos y desafíos de la globalización con herramientas ofimáticas que permitan su autosatisfacción, y por qué no, atender de forma efectiva las demandas desde el interior de la organización para la cual trabaje.

Ahora bien, en la cotidianidad, el ocio constituye relaciones dialógicas con otros campos además del trabajo, tales como la educación, política, economía, el lenguaje, salud, arte, ciencia y naturaleza, entre otras dimensiones de la realidad social contemporánea, compartida, siendo parte integrante y constitutiva de cada sociedad. Por lo tanto, cobra sentido e interés lo que Gomes (2014), plantea, al expresar que el ocio se configura, conforme a las peculiaridades del contexto en el cual es desarrollado, este implica una oportunidad a la cultura, en el sentido lógico, abstracto, configurativo y transformativo de prácticas culturales vivenciadas lúdicamente por personas, grupos, sociedades e instituciones. 
Sin embargo, para lograr visualizar los nexos entre gamer y ocio, los autores del presente manuscrito, convienen en la necesidad de esclarecer el contenido del gamer, el cual pudiera generar cambios tanto en la forma, como el fondo de las estrategias organizacionales, vinculadas a los planes, programas y políticas orientadas al desarrollo integral del talento humano, aplicadas en la gerencia ejercida por transformacional, asumiendo el liderazgo, quienes gestionan al talento humano, tejen los hilos al pináculo de la pirámide invertida. Con referencia a lo anterior, desde su lugar Buitrago y Vera (2019), formulan los siguientes planteamientos:

Gamer, no es sino un anglicismo que adquirió relevancia al finalizar las décadas de los 70’s e inicios de los 80’s y que posteriormente experimentó gran evolución en los años 90’s, con el auge de los dispositivos electrónicos, así como el desarrollo de las plataformas de interacción social múltiple a principios del año 2000 (p. 29).

Asimismo, en opinión de Sandoval (2017), la sociedad de hoy, impregnada de mecanización y automatización en sus diversos ámbitos, propicia que las personas no necesiten hacer grandes esfuerzos para movilizarse, estudiar, trabajar y aún incluso desenvolverse en el hogar. Esto hace que el ser humano disponga cada vez más de tiempo libre, ese tiempo que tanto interés ha generado desde la segunda mitad del siglo XX. Reconociendo al ocio como un intervalo cuya finalidad pueda ser, entre otras, el descanso, relajación, huida de la civilización y rutina, la responsabilidad, el disfrute de la naturaleza, ejercicio físico, interacción con la familia, el altruismo, diversión, recreación, esparcimiento, el desarrollo del sujeto o el encuentro consigo mismo. Por su parte, Buitrago y Vera (2019) plantean:

En los entornos organizacionales los gamers, son considerados un grupo social inmerso y apasionado por los videojuegos, lo que por años fuera considerado menos que un pasatiempo infantil, erigiendo así formas de discriminación sobre los profesionales que aun siendo instruidos, eficientes y productivos, sean etiquetados como videojugadores y solo eso. Sin embargo, al reconocer que las TIC se han involucrado en casi todos los aspectos de la vida diaria, especialmente en aquellas que atienden el entretenimiento para adultos y jóvenes, contemporáneos, se visualiza la oportunidad para que quienes las dominen, desde sus primeros años de vida, ejerzan un rol protagónico de gran importancia; para el curso de los eventos al interior del contexto profesional que integren los gamers (p. 33).

De allí, que se haya considerado al gamer como un proceso de interés interdisciplinario, además de reconocer en él, artistas de carácter humano fundamentalmente integrativas, que le vinculan a otros grandes procesos, advirtiendo que hasta entonces, solo ha sido abordado desde la perspectiva psicosocial, como aquel hombre adepto al uso, disfrute y recreación con videojuegos, algunas veces incluso estigmatizado por ello. Sin embargo, en este escrito, ha sido elevado como forma de comportamiento humano evolutivo, que pudiera favorecer la gestión estratégica administrativa y operativa en organizaciones, es decir, se le está adjudicando, un matiz típico de los fenómenos abordados por la psicología industrial organizacional, dando lugar al ocio, como el escenario ideal para su progresión y prolongación, una categoría científica formal para las ciencias sociales, administrativas y económicas. 


\section{Fundamentación TeóRICA}

En opinión de Acosta (2017) y Del Cioppo y Bello (2018), la sociedad contemporánea se construye sobre una serie de cambios y transformaciones constantes, los cuales han desmontado esquemas sociales clásicos y arcaicos, así como los más elementales sistemas culturales profundamente reconocidos, teniendo esto como consecuencia directa las modificaciones en los patrones de comportamientos de los hombres, incluso desde los primeros años de infancia en aspectos como el aprendizaje, interrelación, comunicación, diversión y recreación, todo lo cual refiere al gamer.

Ahora bien, desde su lugar, Sandoval (2017) apunto que durante décadas algunos estudiosos del comportamiento humano en sociedad, han visto al ocio y el tiempo libre como una pérdida de tiempo. Confundiéndoles con flojera, son considerados como algo que se debe combatir con trabajo o deporte y no como una oportunidad para que el individuo crezca y mejore su calidad de vida. Aunque el ocio sea un fenómeno con un amplio espectro de posibilidades, tanto positivas como negativas, el ocio autotélico, un ocio vivido positivamente y como fin en sí mismo, libremente elegido, satisfactorio, adecuado a la edad y las circunstancias, rico y variado, es el marco de referencia para encaminar las distintas acciones educativas de ocio hacia valores y planteamientos fundamentales.

Entre tanto, Gomes (2012) ha referido que lo anteriormente descrito, sucede con ocasión a hechos vinculados con la realidad sociocultural, que por siglos se ha generado en regiones de Centro América, Sur América y el Caribe, donde no son pocos los que se ven obligados a vivir en la pobreza, desigualdad, hambre. Situación de la que se exalta, la significativa estrechez material que abunda en el tercer mundo. Lo anteriormente descrito, fundamenta la idea de que el ocio y todo lo que le configura, constituye no sólo un lujo innecesario, sino también una pérdida de tiempo para la sociedad latina en el mundo. Igualmente, el mismo Gomes (2012) ha planteado que:

El ocio es comprendido como necesidad humana, una dimensión de la cultura, caracterizada por la vivencia lúdica de manifestaciones ilustrativas en el tiempo/espacio social. Las manifestaciones culturales que constituyen el ocio, pueden ser consideradas prácticas sociales vividas como disfrute de la cultura. Entendidas así, las diversas manifestaciones culturales del ocio son saberes, representaciones, expresiones y lenguajes humanos lúdicamente vivenciados, englobando también los instrumentos, objetos, artefactos y lugares culturales que integran la vida cotidiana de comunidades, grupos y personas (p. 1006).

Por otro lado, en opinión de los autores del presente extenso, corresponde reconocer que en palabras de expertos como Roque (2008), sucede que "las décadas de los años ochenta y noventa, dieron apertura a la intensa comercialización del ocio y el tiempo libre, acentuando desde otro ángulo los procesos sociales y comunicativos que subyacen en el ocio" (p. 12). En ese sentido, resulta ineludible exponer de forma sucinta, el origen del ocio como categoría o fenómeno, proceso de interés para las ciencias humanas, sociales, administrativas en general, es decir el momento en el cual la comunidad científica dio un lugar al ocio y el tiempo libre, como evento científico, a lo que el mismo Roque (2008) refiere: 
Fue en la década de 1950, el período en el que comenzó un verdadero auge en los estudios sociológicos sobre el tiempo libre. En 1956 se celebró en Amsterdam el III Congreso Mundial de Sociología, en el cual se formó un grupo internacional de estudios sobre el tiempo libre, que promovió la discusión y edición de múltiples trabajos sobre esta temática. Constituyéndose la World Leisure and Recreation Association, se impulsó la formación de asociaciones regionales y nacionales, la celebración de congresos periódicos, investigaciones y estudios de distinto carácter y su publicación, básicamente a través de su revista World Leisure and Recreation, de amplia circulación, así como merecido prestigio científico internacional (p. 11).

Ahora bien, el ocio como fenómeno de interés social, reviste un conglomerado de elementos de interés científico, los que a su vez se vinculan a otros, para los cuales el primero reviste una o varias oportunidades de desarrollo e incluso prolongación. En su entendimiento, se edifican los esquemas cognitivos, que resultan útiles para comprender diferentes formas de representación del comportamiento humano, de las cuales el destaca el gamer, como arquetipo por el cual se decantó el presente estudio. Con relación a lo anterior, Gomes (2012) plantea lo siguiente:

Por medio de los estudios sobre el ocio y la recreación, pudiera inferirse el alcance del bienestar y la calidad de vida de las personas. El ocio se constituye de acuerdo con las peculiaridades del contexto histórico y sociocultural en el cual tiene ocurrencia, por eso precisa ser tratado como un fenómeno social, político, cultural e históricamente situado. En esta perspectiva, el ocio se llena de un potencial significativo para, a partir de distintos lenguajes, ampliar la comprensión sobre el mundo, la vida misma y la forma en la que esta es vivida (p. 1006).

Igualmente, en su carrera como investigadora, Gomes (2012) obtuvo hallazgos donde se exponía que el ocio o momentos de ocio, no siempre eran percibidos como episodios fructíferos o necesarios para el desarrollo humano, de allí que Gomes (2014), exprese lo siguiente:

Sin duda, las prácticas de ocio vivenciadas en las metrópolis europeas fueron propagados en otras realidades, como en las Américas, donde los amerindios y los africanos esclavizados muchas veces tuvieron que abandonar sus costumbres y deshacer sus comunidades para integrarse al modo de vida occidental hegemónico (p. 1005).

Por ello, culturalmente durante décadas, este fenómeno ha sido considerado como pérdida de tiempo, pues incluso en estas lides, ejerce impacto la miseria que ha pululado en el tercer mundo, como consecuencia material del imperio hegemónico. Con referencia a lo antes planteado, surge el interés en los autores del presente escrito, exponer que representa para los estudios en psicología industrial/organizacional el ocio, como fenómeno de carácter social y humano, en el que los hombres pueden y tienen oportunidad de autoreconcerse, como elementos de cambio al interior del contexto organizacional contemporáneo en Puerto Rico.

En ese sentido, Montes (2016) ha expuesto que en momentos de ocio, pueden desarrollarse diferentes procesos cognitivos, apoyándose en la agilidad neuronal de los gamers, en su rol como trabajadores hacia su potencialización, recordando que ellos se cualifican por configurar pensamientos en red, promoviendo así la socialización 
del conocimiento, favorecida por la inteligencia colectiva como proceso humano. Indistintamente, compartir un lenguaje propio dentro de la jerga gamer, es parte de la identidad, así como de su integración social, que a su vez refuerza la identidad propia del grupo, y un proceso de internalización que tienen los sujetos cuando toman contacto con el entorno del gaming y la gamification.

Conforme a lo anterior, se infiere que en el abordaje del ocio como teoría de apoyo, se logra comprender el comportamiento del gamer en las nuevas realidades del trabajo. Es menester orientar la percepción de las diferentes cualidades, que en su tiempo libre este sujeto puede desarrollar, potencializar e incluso capitalizar, aprovechando las experiencias adquiridas por medio de las TIC, ello atiende al hecho de que, aun en los escenarios donde desde la mirada del otro, el pensamiento no pudo ir muy lejos, al interior de cada persona pueden estarse desarrollando las mejores ideas, precedidas por necesarios espacios en blanco con lugar al desarrollo de la epoché.

Finalmente, surge así el interés por desarrollar un cuerpo escrito documental, donde se expongan laudos epistémicos que logren esclarecer, que tan profundo así como fecundo, puede resultar el ocio, como oportunidad al desarrollo de la epoché, reconociendo que en tales momentos, en los que el gamer pudiera no estar haciendo nada o al menos pareciera ser así, tendrían lugar diferentes fenómenos, que atiendan la posterior satisfacción de intereses o necesidades diversas o bien y por qué no, erigir al ocio como el escenario ideal del cual emanarían muchas de las grandes ideas, que trajeran consigo la prolongación gerencial, amén de su éxito, ofreciendo espacios a un conglomerado de otros tantos procesos, como la comunicación en masa, socialización del conocimiento, inteligencia colectiva y la gerencia de la felicidad.

\section{MÉTODOLOGÍA}

\section{Diseño}

Se trata de un artículo de tipo documental, en el cual los autores exponen sus perspectivas sobre el comportamiento humano cualificado como gamer, contextualizado en organizaciones contemporáneas en Puerto Rico, relacionándose con otros procesos inherentes al comportamiento humano y organizacional, pero con un giro sui generis; donde se le vincula al ocio productivo, advirtiendo los elementos fundamentales que le configuran. Dichos planteamientos se sustentan en el enfoque analítico y documental, sobre este tipo de manuscritos; los autores Hernández y Buitrago (2017) opinan que:

La investigación (...) documental resulta de gran utilidad, tanto para los investigadores como para los futuros lectores, pues en esta no solamente se comparten los puntos de vista de distintos autores (...), sino que también se manifiesta como un constructo claro de convergencia de ideas, en donde confluyen personalidades, pensamientos y reflexiones pertenecientes a diversas personas de distintas líneas de tiempo (p. 71).

En ese sentido, es importante dejar claro que el extenso en cuestión se apoyó en el método bibliográfico y diseño bibliográfico, con referencia a ello, los mismos expertos, Hernández y Buitrago (2017), exponen lo siguiente: "Este diseño se genera a través de una compleja investigación teórica, con la cual se concrete un entramado doctrinal, 
que sirva como base para realizar un constructo, donde puedan encontrarse respuestas acertadas a las inquietudes planteadas por los investigadores" (p. 70).

Con relación a las investigaciones documentales, expertos como Arias (2012), indican que la investigación documental es un proceso basado en la búsqueda, recuperación, análisis, crítica e interpretación de datos secundarios, es decir, los obtenidos y registrados por otros investigadores en fuentes documentales, impresas, audiovisuales o electrónicas.

Por otro lado, el manuscrito fue desarrollado con apoyo al método científico, de allí que resulte necesario tomar en cuenta lo que con referencia al método bibliográfico; tienen que decir autores como Botero (2003), quien ha referido lo siguiente: "La investigación documental o bibliográfica, configura un modelo que busca, ante todo, posibilitar una investigación reflexiva-analítica, dejando dé lado o en segundo plano acepciones matemáticas, demostraciones empíricas y demás” (p. 111). En la elaboración de un artículo documental, las fuentes de información formal son de tipo neurálgico, pues en ellas reposan los contenidos que dan vida al documento en cuestión. Por ello, Botero (2003) plantea:

La bibliografía no es un simple listado de textos a ser consultados, sino que llega a constituirse como una técnica investigativa que se ocupa de reunir, organizar, difundir y recuperar, a través de un riguroso trabajo, la información que se encuentra en las formas impresas de transmisión del conocimiento. De esta manera, la bibliografía va mucho más allá que un simple listado de textos: es un ejercicio disciplinado para encontrar información en los documentos. La bibliografía es una técnica viva en la investigación documental (p. 112).

\section{Documentos revisados}

El presente artículo ha condensado parte del contenido más relevante, que a juicio de sus autores ha sido publicado en los últimos años con relación al ocio vinculado al gamer. Conforme a ello, sus autores evaluaron cada uno de los artículos que como base científica, constituyen la fuente primordial del conocimiento nuevo. Todo lo anterior, fundamentó las bases para el desarrollo de los planteamientos formulados en el presente estudio. Asimismo, se realizó la auscultación del comportamiento humano y organizacional, desde una perspectiva sofista, sí, pero también desde los laudos epistémicos propuestos por expertos en disciplinas como la psicología industrial organizacional.

En este sentido, el desarrollo del extenso embarga diversos propósitos de distinta índole, entre los que se cuentan presentar la postura de sus autores desde el pensamiento crítico y reflexivo, sobre dos de los procesos que en la contemporaneidad han encontrado lugar al interior de las organizaciones. Al respecto, Sabino (2006) plantea que las investigaciones de corte descriptivo se proponen conocer grupos homogéneos de fenómenos para poder establecer así una descripción única de sus características y el ambiente.

Al mismo tiempo, Gallardo y Camacho (2008) expresan que las investigaciones de corte descriptivo; ostentan como propósito exponer las características de una comu- 
nidad seleccionada para su estudio; en ello se realiza un sondeo, con el que se pueda concretar alguna forma especifica de acercamiento a esta y poder elaborar un diagnóstico, bien sobre el contexto, fenómeno o situación problema que haya llamado la atención del investigador científico.

Al respecto, desde su experiencia autores como Sabino (2006) y Hernández, Fernández y Baptista (2006) expresaron que las investigaciones descriptivas, se proponen conocer grupos homogéneos de fenómenos para establecer una descripción de sus características. Por otro lado se afirma que la investigación descriptiva busca especificar las propiedades importantes de las personas, grupos, comunidades o cualquier otro fenómeno que sea sometido a análisis.

\section{Técnicas e Instrumentos}

El diseño de investigación del presente artículo se apoyó en la revisión de referencias bibliográficas, con abordaje al gamer y al ocio como fenómeno y escenario, respectivamente; donde el primero puede echar mano de sus recursos internos en el segundo, para generar así procesos novedosos a favor del desarrollo personal y organizacional. A los fines de lograr su concretación, como documento de interés científico, fueron seleccionados diferentes archivos documentales, de naturaleza escrita en su versión física y electrónica, de los cuáles su mayoría serían artículos indexados, publicaciones científicas, hechas a través de revistas universitarias, digitales y arbitradas.

Ahora bien, en opinión de Tamayo y Tamayo (2007) es importante dejar claro que, teniendo en cuenta que las investigaciones descriptivas comprenden, valga la redundancia... la descripción, registro, análisis e interpretación de la naturaleza y la composición de los procesos o fenómenos, al mismo tiempo, en este tipo de investigación, se trabaja sobre la realidad de hechos y su característica fundamental, es presentar una interpretación correcta. Del mismo modo, se reconoce como técnica aprovechada en el presente estudio a la documentación, pues en su elaboración, se tomó como fuente principal el contenido que reposa en distintos archivos escritos de interés científico; sobre los cuales Rojas (2011) se refiere de la siguiente forma:

En general, las fuentes de información utilizadas en la investigación documental, se denominan genéricamente: Unidades Conservatorias de Información, y se trata de personas, instituciones, documentos, cosas, bibliografías, publicaciones, Estados del Arte, Estados del Conocimiento, Tesis, Bases de datos, fuentes electrónicas situadas en la red web, etc. cuya función es la de almacenar o contener información (p. 281).

De acuerdo con lo planteado durante todo el extenso, se deja claro que la documentación, como técnica aplicada por los autores en el presente artículo, comprende un proceso cognoscitivo complejo, pues no solamente se apoya en la búsqueda, selección y discriminación de archivos escritos, con contenidos de interés para la investigación original de la que se ha desprendido este extenso, sino también, comprende el reconocimiento de contenidos innovadores que permitan generar una obra literaria, que enriquezca el ámbito científico y académico, desde la episteme y no desde la doxa. Del mismo modo, se le atribuye al ya referido Rojas (2011), lo siguiente: 
La Técnica de investigación documental, también conocida como documentación, se aplica a algunos tipos de documentos escritos: libros, artículos en revistas, reportes de entrevistas, notas de clase, registros de observación directa. Entre estas se cuentan las técnicas de investigación documental de Recuperación: Interpretación hermenéutica (p. 281).

De acuerdo con lo descrito anteriormente, se fundamenta aún más que la naturaleza del extenso es documental, e igualmente se reitera con apoyo a lo expuesto por Sierra (2010), que la técnica aplicada fue la documentación, sobre los documentos seleccionados para el desarrollo del proceso hermenéutico, de allí, que se trate de una investigación documental. Esta técnica, escogida por los autores del manuscrito, consiste en el análisis de informaciones basadas en documentos, los cuales se organizan para dar origen a un nuevo conocimiento, tomando como referencia escritos válidos para el estudio.

Por ello, se aplicó la técnica de investigación documental en la contrastación de archivos, cuyo contenido representó gran significancia para los autores del extenso, al tiempo que fungieron como fuente principal del conocimiento; generado por las ciencias sociales y administrativas esencialmente. Por otro lado, Rojas (2011) afirma lo siguiente: "La técnica de investigación científica es un procedimiento típico, validado por la práctica, orientado generalmente - aunque no exclusivamente- a obtener y transformar información útil para la solución de problemas de conocimiento en las disciplinas científicas" (p. 278).

A la luz de las ideas anteriores, se generó un nuevo documento con el que sus autores, lograron materializar algunos de sus más determinantes laudos, referidos al gamer y al ocio como fenómenos de interés científico, para disciplinas como la psicología industrial organizacional, con el cual se concretó el presente artículo en el que reposan los contenidos de su autoría. Asimismo, se considera, entonces al manuscrito como parte de la propuesta teórica original que finalmente generó la investigación principal de la cuál este se desprende.

Ahora bien, resulta fundamental explicar tales o cuales, fueron los instrumentos aprovechados en la elaboración del extenso en cuestión; entre los que se cuentan la matriz bibliográfica, de uso muy común, por no decir habitual en investigaciones de orden documental, más aún, si estas abordan fenómenos de naturaleza social y humana, como es en este caso en específico; de allí, que se advierta la importancia de aquella, la cual Gómez, Galeano y Jaramillo (2015) desde su lugar plantean:

La matriz bibliográfica comprende un instrumento diseñado en Excel donde se inventariaron todos los textos que conformaron el universo y sobre el cual se aplicaron los filtros de selección. También cumplió la función de reseñar los textos, y lo hizo de forma práctica y asequible (p. 246).

En ese mismo orden de ideas, cabe señalar lo referente a la matriz de análisis de contenido, pues la misma resultó de gran apoyo como instrumento investigativo de carácter neurálgico, no sólo por la naturaleza del estudio, sino también por el tipo de artículo que se presenta en esta oportunidad. Por ello, conviene tener en cuenta aquello que desde su experiencia, Andréu (2002) ha planteado: "Esta se basa en la lectura textual o visual, como instrumento de recogida de información, lectura que a 
diferencia de la lectura común, debe realizarse siguiendo el método científico, es decir, debe ser, sistemática, objetiva, replicable, valida” (p. 2). Para Gómez et al. (2015) sucede que:

La matriz analítica de contenido comprende un instrumento diseñado en Excel donde se relacionaron los textos de la muestra, escritos en vertical, con las categorías de análisis, escritas en horizontal. De cada texto se extrajeron todos los párrafos o frases donde se desarrolló un tema relacionado con alguna de las categorías y se ubicó en la escuadra. También contó con la bibliografía y las observaciones de cada texto de una forma organizada que facilitó la lectura lineal y transversal (p. 426).

\section{Procedimientos}

Para el desarrollo del presente artículo, inicialmente los autores se avocaron a la búsqueda, discriminación, selección y sistematización de diferentes fuentes de naturaleza documental, específicamente de tipo escrito, en la que reposaran las teorías referidas al gamer y al ocio, pero desde el enfoque andragógico, ya que en este manuscrito se procuró desarrollar un constructo que diera cuenta de la dinámica que encontraba lugar, con la condensación de ambos procesos. Al mismo tiempo, cabe destacar que gran parte del conglomerado de archivos encontrados, estuvieron orientados al estudio de los mismos, desde la vorágine social y humana, pues dichos fenómenos son usual objeto de estudio de las ciencias sociales y humanas.

Así pues, se procedió a la búsqueda de fuentes documentales se interés, inicialmente en los principales índices académicos: Publindex, REDIB, DIALNET, RedALyC, SciELO, Latindex, utilizando como criterios de búsqueda palabras relacionadas a cada categoría, entre las que se contaron: gamer, ocio, videojuegos, TIC, entre otras; de donde emergieron gran cantidad de artículos científicos que con abordaje al gamer y al ocio, permitieron configurar una base de datos lo suficientemente robusta como para iniciar el procedimiento investigativo. Conforme a ello, por su parte, autoras como Martín y Lafuente (2017) plantean lo siguiente: "La revisión bibliográfica constituye una etapa esencial en el desarrollo de un trabajo científico y académico. Implica consultar distintas fuentes de información, catálogos, bases de datos, buscadores, repositorios; y recuperar documentos en distintos formatos" (p. 152).

Entre tanto, es preciso reconocer que durante el momento heurístico del proceso investigativo, se procedió a la búsqueda, selección y discriminación de los documentos que por su contenido, resultasen de interés profundo para la investigación, es decir, que ofreciesen el mayor cúmulo de información indubitada posible, para así lograr enriquecer el umbral epistémico del presente estudio.

Ahora bien, concretado, culminado y perfeccionado el procedimiento anteriormente explicado, se procedió al análisis e interpretación crítica y dialéctica, de los contenidos elegidos para la elaboración de dicho constructo, lo cual se denomina momento hermenéutico, para así finalmente se generar un artículo de interés académico y científico verdadero. Con respecto a ello, Arráez, Calles y Moreno (2006) explican lo siguiente: "La interpretación viene a identificarse con la comprensión de todo texto cuyo sentido no sea inmediatamente evidente y constituya un problema, acentuado, 
por alguna distancia histórica, psicológica, lingüística, que se interpone entre nosotros y el documento" (p. 173).

Así pues, en el momento hermenéutico del proceso investigativo, los autores de este extenso, tuvieron la oportunidad de profundizar en el análisis y reflexión de la temática abordada, teniendo en cuenta que tanto el gamer como el ocio, fueron integrados, es decir, se dio cuenta una figura más universal y compleja, ya que se escenificó al primero dentro del segundo, como escenario mayormente prolífico en el contexto laboral contemporáneo. Respecto a ello, autores como Arráez et al. (2006) refieren lo siguiente: "El hermeneuta es, por lo tanto, quien se dedica a interpretar y develar el sentido de los mensajes haciendo que su comprensión sea posible, evitando todo malentendido, favoreciendo su adecuada función normativa y la hermenéutica una disciplina de la interpretación” (p. 173).

Con relación a lo planteado en este segmento y a los fines de sustentar la distinción que los autores han sostenido sobre el presente artículo; resulta preciso atender el tipo de investigación que en este se describe, sobre la cual expertos en la indagación de naturaleza documental. Desde su lugar, Buitrago y Vera (2019) afirman lo siguiente: "Las investigaciones documentales con diseño y método bibliográfico, se caracterizan como formales, teóricas y abstractas, pues la información recopilada, recabada, registrada, analizada e interpretada en su desarrollo y completación, fundamentalmente se obtiene de fuentes documentales y otros soportes de tipo digital” (p. 48).

\section{Resultados y Discusión}

\section{Ocio y tiempo libre: \\ Complejidad en la existencia del trabajador puertorriqueño contemporáneo}

El ocio productivo, conforma un conglomerado de procesos blandos, donde interviene no solo la acción, sino también la voluntad, de allí que se le considere como una suerte de proceso volitivo, donde quien intervenga en el, lo hace con el ánimo evidente o no, de generar figuras de interés propio o común en su divertimento, aún incluso en el contexto laboral, donde el gamer reconozca espacios de acción recreativa. Advirtiendo los eventos descritos en el segmento anterior, es necesario atender: ¿Que hacen los trabajadores cuando aparentemente están desocupados?; en ese sentido, se visualiza al gamer como elemento activo de las dinámicas socioeconómicas, así como del mercado laboral puertorriqueño: ¿Qué hacen? — ¿Qué ocurre en esos momentos de ocio? — ¿Cuáles o tales son sus logros? — ¿Cuál es el alcance de estos logros? Con relación a esto, Gomes (2014) plantea lo siguiente:

En las sociedades capitalistas neoliberales, la forma más inmediata de abordar la temática del ocio expresa su vinculación con el trabajo, lo que es evidente, porque el aspecto relacional, pone en evidencia las fronteras existentes entre ambos. Pero esto es comprensible hasta cierto punto, ya que lo más común es la reducción del ocio a una relación dicotómica, donde su existencia es justificada solamente por las necesidades e intereses del trabajo productivo, lo que representa uno de los principales aspectos del concepto moderno de ocio (p. 11). 
Por ello, corresponde esclarecer cuál es el contenido de tales escenarios y que fenómenos encuentran oportunidad en ellos para su desarrollo efectivo, teniendo en cuenta que no es fácil para el gamer ejecutar sus acciones o actuaciones en un contexto que presente limitaciones ofimáticas, pues en el orden administrativo y operativo, sus habilidades y destrezas son demostrables con apoyo a recursos tecnológicos; no así, con respecto a sus cualidades como estratega, ya que este conglomerado de procesos neuronales tienen origen en su mente, extendiendo así, sus aportes al equipo de trabajadores con quienes labore. Así pues, sin llegar a extender el manuscrito en un manifiesto idealista, al menos desde una perspectiva humana, se aspira auscultar: ¿Qué hacen en momentos de ocio los trabajadores/gamers... que genere algún beneficio para su autorrealización personal, profesional, laboral?

Igualmente, vale destacar, en el desarrollo y ejercicio de su personalidad, los gamers exponen cualidades disímiles entre sí, inherentes a cada una de las diferentes inteligencias con las que pudieran contar. Con ocasión a ello, el contexto laboral se erige como el espacio ideal, donde logran prolongar su estatus quo, al mostrarse como profesionales con suficientes conocimientos ofimáticos, con los que distinguirse entre compañeros de trabajo, generando desde su lugar mayores aportes que el resto, en procesos y procedimientos que necesariamente deban ser informatizados. Según Montes (2016), los gamers han generado cambios en el comportamiento humano, así como también la tecnología en conjunto con la sociedad, ha ocasionado una evolución significativa, al punto que esta última se ha vuelto más tolerante hacia los gamers.

Ahora bien, en el contexto laboral contemporáneo, los trabajadores puertorriqueños que se autocalifiquen como gamers, afrontan diferentes escenarios, donde corresponde ejerzan disímiles roles, para con ello resolver de forma inteligente, práctica y efectiva cualquier situación de la que hagan parte, entre estos se cuentan: comunicación, socialización, ejercicio profesional, acoso laboral, informatización de procesos, conflicto de intereses, toma de decisiones, trabajo de campo, digitalización de los contenidos, programación de estructuras, entre otras tantas.

En ese sentido, corresponde plantear el desarrollo de algunos procesos, en los que como trabajadores, corresponde participar a los gamers puertorriqueños, de los cuales, conforme a la opinión de los autores, destaca por su naturaleza, así como también alcance: la comunicación. Ya que, como actores sociales, los gamers deben comunicarse de la forma en la que según sus cualidades y circunstancias del contexto lo permitan. Con relación a ello, Buitrago (2016) afirma: "Los fenómenos del comportamiento humano encuentran su apoyo en procesos que además de complejos, son demorados y profundos" (p. 74).

Sobre la base de las ideas anteriores, Hernández y Buitrago (2017 afirman: "Desde los albores de la humanidad, el proceso comunicacional y todos sus referentes se encuentran incrustados de manera intrínseca en el hombre debido a su naturaleza de carácter social" (p. 62). Por ello, resulta muy bonito, tener en cuenta el valor de los intangibles, por todo aquello que generan en si mismos; Castellano, Hernández y Caridad (2017) plantean: 
La importancia del talento humano no se sustenta solo en la gestión, sino que también abarca las decisiones que toma la empresa, orientándose en la optimización de recursos para mejorar el desempeño laboral, creando valores corporativos con miras a desarrollar el capital humano, y que este sea el objetivo fundamental de la empresa" (p. 240).

Igualmente, se infiere que en momentos de ocio, los gamers se comunican con otros gamers, así como también lo hacen con jugadores, que no califiquen como gamers por su conducta demostrada hacia los videojuegos, los cuales reciben la denominación de usuarios; estos por su parte participan en las dinámicas de la vida diaria del gamer, lo mismo como familiar, amigo o incluso compañeros de trabajo. De cualquier modo, los momentos de ocio y tiempo libre, generan interés científico, porque en opinión de los autores del manuscrito, incluso allí, aprovechan el tiempo para generar ideas, pensamientos, impresiones, estén o no practicando con videojuegos, pues los momentos de ocio, pueden y son aprovechados para tantas acciones, como la voluntad del hombre así lo desee.

Con relación a las ideas expuestas, esta claro que aunque el escenario sea virtual o real, el gamer hace lo necesario por lograr su cometido; en ello cobra importancia lo que Caro (2015) expone: "Cada nuevo medio permite y propone al sujeto nuevas formas de relacionarse con el entorno y los otros" (p. 25). Así pues, vale abordar escenarios donde estos se comunican, para comprender de qué manera tales conversaciones generan algún elemento a favor de las dinámicas entre gamers, con relación a otros gamers o usuarios. La comunicación es un proceso humano fundamental en la sociedad contemporánea (Barragán, García, Meza, Mercado \& Olarte, 2020), por lo tanto, los autores se han decantado por esta como el fenómeno más dúctil, con el que se puede profundizar en lo que atañe a las cualidades y competencias con las que los gamers cuentan.

Con atención a lo descrito en párrafos anteriores, los autores Hernández y Buitrago (2017) han afirmado que "las organizaciones han tomado un rumbo más humanizado y flexible, donde uno de sus principales objetivos es generar resultados orientados al bienestar social, personal y psicológico de los trabajadores" (p. 62). Lo anterior, se ha visto reflejado en la gestión gerencial contemporánea, favoreciendo procesos como la motivación y satisfacción laboral, ante lo cual, Parra, Durán, Romero \& Patiño (2017b) plantean lo siguiente: "Por medio de la calidad de vida laboral, el ser humano puede construir, pensar, inventar, desarrollar nuevas formas de convivencia, sistemas económicos, políticos, sociales, empresariales, entre otros" (p. 178).

Conforme a lo anterior, otros autores como Hennessey \& Rincón (2018) afirman: "Comunicarse e interactuar es indispensable para establecer relaciones entre las personas, para coordinar las acciones y tareas a realizar en conjunto y facilitar la evolución y desarrollo de individuos, grupos y comunidades" (p. 78). Lo planteado se relaciona con las diferentes destrezas; que los gamers exhiben en los procesos de comunicación e interacción que sostienen con éxito, tanto con otros usuarios como entre gamers. Por ello, reviste importancia analizar y reflexionar en los eventos y/o fenómenos, que se suceden en momentos de ocio y tiempo libre, tal es el caso de la comunicación entre gamers. Llama la atención, porque esta es un proceso común para los seres humanos (Barragán et al., 2020), que conviven e interactúan constantemen- 
te, lo cual erige a la comunicación como escenario de gran interés, si de auscultar el comportamiento humano se trata.

En ello, surgen como elementos potencializadores: espacio y tiempo, donde el gamer lograría concretar además de la comunicación, un sinnúmero de procesos, en los que se verían involucrados por medio de la telepresencia, fenómeno contemporáneo con origen al auge del internet, redes sociales y otras plataformas de comunicación y socialización, así como también las TIC en general. Igualmente, lo anterior se orienta a la consecución de procesos psicosocialmente complejos, donde en momentos de ocio los gamers como profesionales/trabajadores, tendrían la oportunidad de generar ideas a favor de los procesos con lugar en la realidad laboral. A la luz de las ideas anteriores, puede visualizarse cuán importante resulta abordar al tiempo libre y los momentos de ocio como fuente de conocimiento. Conforme a lo anterior, Roque (2008) expone lo siguiente:

Los primeros análisis registrados sobre el tiempo libre y el ocio en el marco del pensamiento filosófico occidental, fueron realizados en la Grecia Helénica y alcanzaron una sistematización posterior en el Imperio Romano. Con el término SKOLE Platón y luego Aristóteles, designaban un estado o proceso que con posterioridad ha sido comúnmente conocido por "ocio" y "tiempo libre" en español, "leisure" y free time" en inglés y "loisir" y "temps livre" en francés. Platón, abordó al ocio como un elemento de apoyo a sus concepciones sobre la organización ideal de la sociedad. En toda su obra, pueden encontrarse los sentidos más significativos que asignaba a ese término y que revelan un nivel de reflexión que aún en nuestros días no es común por lo avanzado de las ideas (p. 5).

Según Sandoval (2017), los conceptos de ocio y tiempo libre, están muy relacionados y se refieren a una amplia gama de posibilidades de acción del individuo. Lo anterior permite inferir entonces, que desde hace décadas al ocio y los momentos de ocio se les atribuyó interés social y humano, además deja claro que bien constituye un conjunto de escenarios de acción fructífera para el hombre contemporáneo y jamás refiere a la idea del mismo como vagancia o pérdida de tiempo. Por su parte, Roque (2008) continúa planteando, cuán interesante resulta observar cómo en su herencia filosófica y reflexiva Platón, dejo muestra de sus ideas sobre el ocio, y la importancia de este en el desarrollo de la personalidad y el ejercicio de esta. Relacionado a lo expuesto el mismo autor afirma:

Al retomar los planteamientos sofistas de Platón, no se debe incurrir en el error de separar radicalmente al trabajo del ocio, sino antes bien, sostuvo su unidad. Más aún, defendió la tesis de que el ocio, debía existir en función del trabajo, como una de sus condiciones. Tal concepción, se sustentaba en la estructura social de su época, cuyos roles legitimaba la sociedad esclavista: en las ciudades griegas, quedaba para los ciudadanos las diversas formas de participación en la vida política, educación, filosofía y arte, o en un plano inferior, en el comercio o artesanía, mientras el trabajo productivo esencial descansaba sobre los esclavos (Roque, 2008, p. 5).

En ese sentido, es preciso indicar que los estudios cuyo corte humanista versan sobre el ocio y tiempo libre han sido orientados hacia la exégesis del pensamiento vivo. Por ello, Roque (2008) expone: "Desde un ángulo cuantitativo, es posible afirmar que el empirismo, continúa siendo la plataforma metodológica de la mayor parte de 
los estudios contemporáneos sobre el tiempo libre, salvo focos y autores que han expresado enfoques metodológicos superiores" (p. 10).

Ahora bien, las circunstancias económicas y políticas en la sociedad puertorriqueña contemporánea, aunadas al imperio del gobierno de los Estados Unidos, pudieran determinar tales o cuáles son las necesidades propias, de quienes hacen vida útil y ejercen su profesión en el Área Sur de Puerto Rico, así como también la forma en que estas sean satisfechas, advirtiendo su matriz sociocultural, la cual es totalmente diferente hoy día, de lo que fuera de dos a tres décadas atrás.

Conforme a lo anterior, Parra et al. (2017), plantean lo siguiente: "El trabajo es la actividad que le proporciona al individuo la posibilidad de alcanzar el desarrollo y bienestar laboral, social y familiar, el cual se torna también en una condición propia del ser humano para su pleno desarrollo" (p. 178). Considerando al trabajo como espacio donde los gamers se recrean a sí mismos, conviene tener presente lo que Castellano et al. (2017) en su momento plantearon:

El talento humano se percibe como el potencial a través del cual se logra la eficiencia y eficacia de la organización, por ende se les debe proporcionar los métodos para optimizar su utilización y elevar su compromiso; ello permitirá el aprovechamiento de sus capacidades, de sus potencialidades (p. 240).

De acuerdo con lo anterior, corresponde exponer que lugar ocupa el trabajo como hecho social en la sociedad puertorriqueña, atendiendo, cuestiones de naturaleza política, económica e incluso cultural: advirtiendo la transculturización de la cual ha sido objeto la nación boricua. Para Sabariegos (2011) sucede que, "el trabajo pasa a ser sustancial del sistema capitalista como instrumento necesario para la producción de riqueza, convirtiendo el salario en la mediana que delimita lo que es trabajo y aquello que no lo es" (p. 6).

Con relación a lo descrito en el párrafo anterior, se reconoce en el trabajo el contexto donde muchos gamers encuentran oportunidades a la prolongación de sus conocimientos, no sólo aquellos relativos al uso de recursos ofimáticos, también a tantos otros que como actores del nuevo orden ostentan; teniendo en cuenta que los innumerables fenómenos globales, generan el impacto suficiente como para deconstruir su realidad. Ante lo anterior, autores como Buitrago y El Kadi (2019) afirman lo siguiente: "En la actualidad los factores externos incluso más allá de las fronteras, ejercen algún tipo de influencia sobre la realidad social y política en cada país" (p. 157); lo cual se ve reflejado en el mercado laboral, la realidad interna en organizaciones contemporáneas es descrita por Parra, Durán, García y Ramírez (2017a) de la siguiente forma:

En este ámbito, las organizaciones se ven comprometidas a proporcionar acciones para promover en su personal el desarrollo de su capacidad social en lo que respecta a motivación, remuneración, incentivos o recompensas para impulsar al empleado a dar su máximo rendimiento de manera espontánea (p. 69).

De acuerdo con los planteamientos anteriormente esbozados, se fortalece la premisa de que la fuerza humana de trabajo, instruye el núcleo energético que dinamiza los procesos tangibles e intangibles que dan vida a la organización; en ese sentido, 
cabe mencionar de que forma algunos autores consideran, elevan e incluso empoderan el rol ejercido por trabajadores y colaboradores, entre los que a los ojos de quienes redactan este manuscrito se cuentan los gamers.

De conformidad con lo antes descrito, Parra et al. 2017b) plantean: "Hoy ese valor agregado lo añaden las personas, su talante, su manejo, su forma de hacer las tareas en las empresas; cada día esta diferencia visibiliza organizaciones y con ellas la gente que lo forja" (p. 177). Por su parte y con respecto a lo indicado, Castellano et al. (2017) afirman:

Se hace necesaria la orientación y reestructuración de las organizaciones hacia la excelencia de los procesos de trabajo, lo cual implica la formación capaz y comprometida con el éxito de la empresa, en cada área que la conforma, desde el nivel gerencial hasta el operativo (p. 246).

Así pues, se logra reconocer que si bien es cierto el capital humano, que conforma las filas de trabajadores de una organización, comprende el mayor recurso con el que está puede contar, es también muy real, que este mismo, requiere contar con un nivel de instrucción ofimática suficiente como para poder generar beneficios de cualquier tipo a favor de la organización para la cual trabaje; en ese sentido Prieto, Céspedes, Palacios y Paz (2018) plantean lo siguiente:

Los colaboradores representan el talento humano que da vida y acciona las estrategias desde sus ideas y actitud en cualquier organización, los cuales, generalmente, asumen su participación mediante roles: directivos, supervisores, operarios, asistentes y apoyo logístico; dependiendo de la naturaleza institucional (p. 13).

Por otro lado, surgen planteamientos tales como los propuestos por Buitrago (2016), quien ha manifestado que "las organizaciones persiguen encontrar en sus filas, líderes que se adecuen a sus necesidades y aspiraciones, lograrlo no sólo es costoso, complejo y extenuante, también suele ofrecer oportunidades donde el desconcierto o la especulación trunquen los procesos de búsqueda" (p. 77). Lo anterior, esta vinculado con la necesidad que las empresas de cualquier sector manifiestan, pues echan en falta contar con el talento humano indicado, sobre todo si pertenecen a industrias, que requieren tener entre sus trabajadores, profesionales con niveles de instrucción de mayor nivel que la media, más aún cuando en los tiempos que corren casi cualquier procedimiento se ha informatizado.

A la luz de las aseveraciones anteriores, se infiere que la fuerza humana de trabajo, conforma el contenido intangible del capital organizacional e incluso al pensarlo bien, sin directivos, gerentes, asesores, trabajadores y colaboradores; las organizaciones no serian mas que un proyecto de papel, sin nadie que las eche a andar e impulse su objeto social. Por ello, Hernández y Buitrago (2017) afirman: "En cualquier actividad humana, una persona competentemente profesional es aquella que, aparte de llevar a cabo las tareas de su determinada profesión, es capaz también de manejar tareas simples como, por ejemplo, una buena comunicación con sus colegas" (p. 69). De allí, que se considere al gamer como un empleado que con base a sus conocimientos ofimáticos, pudiera rendir más que lo haría cualquier otro, que no cuente con habilidades tecnológicas. 
En ese mismo orden de ideas, resulta interesante tener en cuenta, que la realidad compartida a nivel global, exhibe elementos estructurales que lejos de renunciar a la informatización, pareciera adherirse a ella con más fuerza cada día; con atención a ello, autores como Gil (2006) han apuntado que: "Las empresas están inmersas en un entorno de cambio permanente y acelerado, donde evolucionan hacia un modelo de producción distinto, que gira en torno al aprendizaje y en que se plantean nuevos retos cada día" (p. 35).

Por esto, un trabajador que exhiba mayor habilidad tecnológica, resulta mucho más efectivo que otro que no cuente con tales prerrogativas, y más allá de ello, le resulta más rentable a la organización, pues cuando los trabajadores no manifiestan destreza en la utilización de recursos ofimáticos, se hace necesario desarrollar programas de instrucción y capacitación, lo cual siempre termina generando egresos eventualmente.

Ahora bien, entre las habilidades con las que cuentan los gamers se tiene al manejo y apropiación de las TIC, como herramientas con las que se sienten cómodos en su aprovechamiento, usualmente a favor de los procesos sistematizados, informatizados y administrativos al interior de las organizaciones contemporáneas en Puerto Rico. Por ello, cobra interés dejar claro que en opinión de Pirela, Prieto, García y Quijada (2018) sucede que: "En el siglo XXI las organizaciones e instituciones compiten por brindar mejores sistemas de información, ofrecer servicios de calidad con tecnología de última generación, este fenómeno se ha presentado con mayor fuerza en los últimos años" (p. 130).

A la luz de los planteamientos hechos en los párrafos superiores, Gil (2006) afirma lo siguiente: "El reto al que se trata de hacer frente es la adaptación y el desarrollo continuo de las empresas para el logro de un éxito sostenido en un entorno como el descrito anteriormente" (p. 34). En ese sentido, expertos en comportamiento humano y quienes han llevado a cabo diversos estudios del ser, Parra et al. (2017b) han expresado:

El hombre es un ser productivo, que vinculado en el ambiente laboral, considera los valores empresariales como un proceso por medio del cual puede cumplir sus propósitos personales, y con ellos impulsa los esperados por la empresa a la cual pertenece" (p. 178).

Según Buitrago y León (2019), al auscultar el comportamiento humano y lograr comprenderle, es más que plausible visualizar diversos elementos vinculantes, necesarios y sobrevenidos entre las ciencias administrativas, humanas y políticas, de allí emergen nuevos planteamientos, acrecentando la variedad de opciones estratégicas y perspectivas desde la tránsdisciplinariedad, apoyadas en el conocimiento como capital intangible. De conformidad con los expuesto anteriormente, Gil (2006) plantea lo siguiente: "En términos económicos, puede caracterizarse la economía contemporánea por tres grandes características: es informacional, es global y funciona en red. Es así, como la información y el conocimiento son las variables decisivas en la productividad y en la competitividad" (p. 36).

Amén de ello, los autores Buitrago y León (2019), citados en el párrafo anterior, coinciden en la importancia de dejar claro que a la fecha, la información se convirtió en un recurso al que se puede acceder con relativa facilidad, con ocasión a ello, cual- 
quier escenario social reviste interés mediático y público. Igualmente, los interesados cuentan con un abanico de opciones para apropiarse de contenidos referentes a ello y al mismo tiempo con apoyo en las plataformas tecnológicas lograr generarlo. En ese mismo orden de ideas, Hernández y Buitrago (2017) han expresado: "En las organizaciones existen caracteres y rasgos excepcionales en la interrelación de las personas que configuran una estructura de comunicación informal dentro de ellas" (p. 61).

Ahora bien, sobre el contexto del que se habla en el párrafo anterior, Buitrago (2019) expone: "Muchas organizaciones echan en falta elementos organizacionales que ofrezcan un cuerpo de agua que refresque los escenarios convulsos del presente" (p. 14). En ese sentido, se visualiza al gamer, como elemento novedoso, diferente, que pudiera generar nuevas oportunidades de gestión de las TIC a favor del talento humano, sí, e igualmente de otros procesos de orden estratégico e incluso operativo; una bocanada de aire fresco que dinamice las estructuras establecidas... si es que ya no lo ha hecho, y por desconocimiento haya pasado inadvertido, pues desde hace varios años, la generación millennials ha integrado las filas de trabajadores activos en el espectro gerencial. Con respecto a ello, Buitrago (2019) plantea:

Corresponde humanizar los procesos, inspirar, motivar, compensar y satisfacer a los trabajadores y colaboradores en el desarrollo de sus actividades laborales o profesionales. Las organizaciones del mundo, están habidas de un personaje capaz de orquestar, no solo las funciones que por cargo deban efectuarse, también pueda estructurar las bases internas de la industria e igualmente les permita competir en el mercado, logrando llevarlas al siguiente nivel (p. 9).

En relación con lo anterior, los autores Parra et al. (2017a) plantean que "es importante para las empresas contar con procesos integrales que orienten el cambio, tomando como línea general la motivación, la cual ha sido relacionada con la productividad y con la eficacia" (p. 69). En ese sentido expertos como Ruz y Lobo (2018) afirman lo siguiente:

Es preciso considerar que los constantes cambios que se suceden en el entorno empresarial, signados por factores asociados con aspectos laborales, clima organizacional, políticos, sociales, entre otros, están en permanente variación, dependiendo de la cultura sobre la cual se realice el análisis, afectándolas de manera directa o indirecta, sin distingo de su actividad principal (p. 111).

Sin embargo, los momentos de ocio o el mismo ocio como fenómeno, reviste caracteres sociales fundamentalmente, pues en su desarrollo adquiere cierta naturaleza humana, cultural, social, la cual atiende procesos del mismo orden, de ahí que comúnmente este haya sido e incluso aun se constituya, como categoría propia del interés por parte de las ciencias y disciplinas sociales. Con relación a ello, Buitrago (2016) afirma que "al pretender dar cobertura a un fenómeno social como el ocio, los aspectos culturales de su entorno serán determinantes en su progresión, desarrollo y éxito" (p. 74). En ese sentido el mismo, Buitrago (2017) plantea lo siguiente:

El proceso de globalización, así como las TIC, estrecharon los lazos entre naciones, acercando aun mas a quienes viven en ellas, todo lo cual gravita en el hecho donde todo cuanto pueda ocurrir en cualquier parte del mundo, tendría quizás alguna forma de impacto en otro sitio, con atención a las relaciones e interconexiones entre países (p. 321). 
Ahora bien, desde su lugar, autores como Sandoval (2009) y Barragán et al. (2020), han referido que los medios de comunicación y las industrias culturales, así como las expresiones de la publicidad comercial, reproducen y socializan en los valores el sistema dominante y amenazan con eliminar el pensamiento y la crítica. Los efectos de esta orientación mediática crean un escenario de cultura cerrado, unidimensional, que propicia una especie de pensamiento único y determina la conducta del individuo en la sociedad, esto incluye: necesidades, intereses, aspiraciones, entre otros.

Conforme a ello, en opinión de Sandoval (2017), al vincular las necesidades con el concepto de ocio, se asume entonces que aunque la motivación intrínseca es una cualidad esencial del ocio, puede pensarse en muchas actividades y experiencias de ocio en las que se actúa por refuerzos extrínsecos, es decir, por el logro de un objetivo ajeno a la mera satisfacción derivada de llevar a cabo una determinada actividad. Al mismo tiempo, Gomes (2014) considera lo siguiente:

Reconocer al ocio y a la recreación como derechos sociales, resulta de gran interés principalmente en los contextos donde las desigualdades sociales son alarmantes y revelan niveles absurdos de iniquidades, como es común en casi toda América Latina y el resto del tercer mundo. De este modo, su realización significa algo profundo que envuelve varios elementos, los cuales posibilitan el surgimiento de interrelaciones dinámicas. Entre estos elementos pueden ser citados: el reconocimiento de identidades/diversidades culturales, la creatividad social y tantos otros, que en conjunto, pueden abrir algunas puertas para la transformación social (p. 9).

Finalmente, con este manuscrito se persigue generar un extenso donde se expongan con fluidez y raciocinio, el interés que en opinión de los autores recae sobre el ocio, como fenómeno de gran contenido cognoscitivo, para lograr comprender al gamer, patrón comportamental representativo, de las generaciones, con origen en la segunda mitad de la década de los 80's y en adelante, manifestación vívida del homo erectus en la Era Digital por la que transita la sociedad hoy día, hasta su transformación como el homo venatus, validando el rol preponderante de los nativos digitales en el contexto social, cultural, laboral, organizacional puertorriqueño contemporáneo, reivindicando al hombre boricua; en una época donde el pensamiento vivo se constituyó eje dinamizador del desarrollo y la prolongación de la humanidad.

\section{Conclusiones}

Conforme a lo anteriormente expuesto, el presente manuscrito, cuenta entre sus aspiraciones, brindar a la comunidad científica, una perspectiva del comportamiento socialmente reconocido como gamer, ajustada a la realidad social compartida en las regiones del tercer mundo; teniendo en cuenta que en los países de la periferia, casi cualquier proceso humano, está permeado de complejidades, limitaciones, condiciones y bloqueos económicos sistemáticos, que son el brazo ejecutor desde la hegemonía de los Estados potencia, advirtiendo que al aprovechar los momentos de ocio, como algo más allá del tiempo libre, podrían emerger nuevos fenómenos, que favorecerían los procesos con lugar en el ámbito organizacional, bajo el impacto de la vertiginosidad tecnológica, el convulso orbe globalizado y la inminente, así como constante transformación del mercado laboral. 
Igualmente, cabe tener en cuenta que lo descrito en el párrafo anterior, se refleja al interior de las organizaciones contemporáneas en Puerto Rico, donde la informatización de los procesos, ha cobrado tanto protagonismo como la capitalización del talento humano. En ese sentido, vale rescatar tales o cuales son los escenarios donde el gamer puertorriqueño, puede aprovechar sus cualidades, habilidades y destrezas a favor de sí mismo y de la organización para la cual trabaje. También, cobra interés reconocer el alcance de sus conocimientos ofimáticos, que les permita aprovechar los momentos de ocio en cualquier contexto administrativo y gerencial, generando así mayores oportunidades de enriquecimiento.

En ese sentido, puede plantearse que con el nivel de instrucción tecnológica suficiente, así como la facilidad en el manejo de recursos ofimáticos, aquellos momentos de ocio productivo, no constituyen una acción inherente a la holgazanería, el tedio o el vicio, son de facto espacios donde el tiempo transcurre para el beneficio del ser, bien sea leyendo, observando el horizonte, ejercitándose, conversando por teléfono, escuchando música, generando ideas, explorando alternativas, creando nuevos modelos de pensamiento y gestión, entre otras tantas acciones que constituyan la recreación, satisfacción y goce.

Ahora bien, cabe decir que desde un enfoque sui generis, el gamer se ha elevado en los últimos 10 años, como la personificación del hombre nuevo, ajustado y robustecido con el uso de las TIC, pues con estas como recursos de acción, favorecen el desarrollo de procesos al interior de más organizaciones, donde diariamente se experimentan transformaciones de diverso orden, que influyen en fenómenos de gran interés para los estudios vinculados a la psicología industrial organizacional, así como otras ramas de la psicología, teniendo en cuenta que estas, se han involucrado en otros tantos contextos: educativo, deportivo, sanitario, artístico, industrial, entre otros.

Sin embargo, en opinión de los autores del presente manuscrito, es menester indicar, que más allá de los entornos donde la tecnología cuente con una ineludible presencia, ante las habilidades y cualidades típicas del gamer: agilidad motriz fina, agilidad visual y óptica, inteligencia lógico-numérica, inteligencia espacial, inteligencia interpersonal, inteligencia social, entre otras tantas, este con facilidad encontraría diversas oportunidades laborales en otras tantas formas, otros escenarios, otros contextos: comercial, cultural, social, deportivo; dicha idea, fue uno de los detonantes, que dieran lugar a la investigación original, de la cual se desprende el escrito en cuestión, intitulada: Implicaciones del gamer en los procesos de trabajo en organizaciones contemporáneas de Puerto Rico.

En ese mismo orden, el propósito de manuscritos como este, es generar interés formal y científico por el ocio como oportunidad de desarrollo para el pensamiento, análisis, reflexión, y epoché para disciplinas como la psicología industrial/organizacional, sobre todo porque al relacionarse con el gamer como actor en el dinámico mercado laboral, informatizado, globalizado, vertiginoso como se presenta en esta época, pero sin obviar los momentos de ocio productivo, como escenario común en las nuevas realidades del trabajo. Tomando en cuenta lo anterior y aprovechando las bases del constructivismo: se propone al gamer en momentos de ocio, como fenómeno de interés científico, para las ciencias humanas, administrativas, económicas, y sociales. 
A la luz de las ideas anteriores, tanto gamer como ocio, son fenómenos que en opinión de los autores, generan oportunidades a la evolución del pensamiento crítico, en tales lides, amén del reconocimiento de nuevas formas de comportamiento en el contexto organizacional. Igualmente, pudiera plantearse que los gamers, se distinguen de otros patrones comportamentales del ser humano, porque en momentos de ocio no pierden el tiempo, ya que si no están creando, están siendo partícipes en el desarrollo de alguna cocreación, lo cual adquiere relevancia y significancia en una época donde la informatización de los procesos se hizo norma, en la que contar con trabajadores habilidosos en el uso de recursos ofimáticos marca gran diferencia, entre la resistencia al cambio y la evolución al éxito.

Entre tanto, durante el desarrollo de la investigación, de la cual se desprende el presente extenso, se reconoció al ocio como la oportunidad, para que el trabajador con habilidades y destrezas en el uso de recursos ofimáticos, encuentre provecho, en aquellos espacios donde al no tener nada por hacer, experimente la necesidad de llenar ese vacío con ideas innovadoras, nuevas propuestas estratégicas, programas, planes, políticas y tácticas de gestión gerencial, sobre todo aquello vinculado a la gestión de personal, potencialización del capital intelectual, monetización de recursos intangibles. Del mismo modo, en el alumbramiento del fenómeno gamer, surgen diversas oportunidades para el desarrollo de otros fenómenos, que dinamicen la realidad poliédrica al interior de las organizaciones contemporáneas en Puerto Rico.

Finalmente, entre los fenómenos indicados en el penúltimo párrafo, cabe mencionar: la socialización del conocimiento, inteligencia colectiva, inteligencia ética colectiva, gerencia de la felicidad, e-commerce, teletrabajo, telepresencia, gaming y gamificación entre otros y en casi cualquier escenario. Conforme a lo anterior, pudiera plantearse que para el gamer, la realidad se percibe como un conjunto de eventos sucedidos y consecutivos, que embargan entre sí un significado relativo y personalísimo, donde adquiere diferentes experiencias, bien de forma individual o compartida.

\section{Agradecimientos y ReCOnocimientos}

Artículo derivado de la investigación intitulada "Implicaciones del gamer en los procesos de trabajo en organizaciones contemporáneas de Puerto Rico"

\section{REFERENCIAS}

Acosta, J. (2017). La experiencia y significados de la práctica de jugar según padres, madres o cuidadores primarios en las habilidades sociales y de comunicación de niños y niñas de 7 a 11 años de edad. [Diserción doctoral]. Pontificia Universidad Católica de Puerto Rico Recinto de Ponce, Ponce, España.

Andréu, J. (2002). Las técnicas de Análisis de Contenido: Una revisión actualizada. Andalucía: Centro de Estudios Andaluces.

Arráez, M., Calles, J. y Moreno, L. (2006). La Hermenéutica: una actividad interpretativa. Sapiens, 7(2), 171-18. Disponible en https://sapiensrevistave.wordpress. $\mathrm{com} /$ 
Barragán, C., García, J., Meza, V., Mercado, M. y Olarte, L. (2019). Responsabilidad social empresarial en medios impresos en los Departamentos del Atlántico y Magdalena (Colombia). Económicas CUC, 41(1), 187-202. https://doi.org/10.17981/econcuc.41.1.2020.Org.3

Botero, A. (2003). La metodología documental en la investigación jurídica: alcances y perspectivas. Opinión Jurídica, 2(4), 109-116. Disponible en https://revistas.udem. edu.co/index.php/opinion/article/view/1350

Buitrago, R. (2019). Liderazgo reformador: una perspectiva cualitativa desde la plasticidad de la industria farmacéutica. Consensus, 3(2), 4-40. Disponible en http:// pragmatika.cl/review/index.php/consensus/article/view/34

Buitrago, R. (2017). Gestión del conocimiento a través del liderazgo femenino como recurso esencial para la competitividad organizacional. CICAG, 14(2), 320-337. Disponible en http://ojs.urbe.edu/index.php/cicag/article/view/136

Buitrago, R. (2016). Liderazgo ético como factor potenciador del marketing social hacia la innovación estratégica en la mercadotecnia moderna. Marketing Visionario, 5(1), 72-87. Disponible en http://ojs.urbe.edu/index.php/market/article/view/2392

Buitrago, R. y El Kadi, O. (2019). Autotomía caudal como herramienta estratégica prospectiva ante un cambio de época en organizaciones públicas. Dictamen libre, (24), 155-177. https://doi.org/10.18041/2619-4244/dl.24.5362

Buitrago, R. y León, L. (2019). Branding personal como eje dinamizador de la imagen política para los candidatos a cargos de elección popular en Venezuela. Cuadernos Latinoamericanos de Administración, 15(27), 1-16. https://doi.org/10.18270/cuaderlam.v14i26.2609

Buitrago, R. y Vera, J. (2019). Desmitificando al gamer: una aproximación ontológica desde el pensamiento crítico ante un cambio de época. Consensus, 3(3), 28-62. Disponible en http://www.pragmatika.cl/review/index.php/consensus/article/view/42

Caro, L. (2015). Relaciones e interacciones parasociales en redes sociales digitales. Una revisión conceptual. Icono14, 13(2), 23-47. https://doi.org/10.7195/ri14.v13i2.853

Castellano, M., Hernández, P. y Caridad, M. (2017). Gestión de talento humano para la generación del compromiso organizacional en empresas constructoras colombianas. En, R. Prieto y A. Cazallo (comp.), Desarrollo Organizacional y Gestión Humana en Contextos Globalizado. Barranquilla: Ediciones UniSimón. 235-268. Disponible en http://hdl.handle.net/20.500.12442/2602

Del Cioppo, J. y Bello, M. (2018). Indicadores de impacto social para evaluación de proyectos de vinculación con la colectividad. Revista Económicas CUC, 39(1), 105-116. https://doi.org/10.17981/econcuc.39.1.2018.07

Gallardo, P. y Camacho, J. (2008). La investigación participativa y su aplicación en el campo social y educativo. Sevilla: Wanceulen.

Gil, J. (2006). La gestión empresarial bajo el enfoque de las organizaciones inteligentes en la sociedad de la información. Negotium, 2(6), 33-54. Recuperado de http:// revistanegotium.org/pdf/6/6Art2.pdf

Gomes, C. (2014). El ocio y la recreación en las sociedades latinoamericanas actuales. Polis, (37), 1-17. Disponible en https://polis.ulagos.cl/index.php/polis/article/ view/1023

Gomes, C. (2012). América Latina, ocio y Geopolítica del Conocimiento. Educación física y deporte, 31(2), 1001-1008. Disponible en https://revistas.udea.edu.co/index. php/educacionfisicaydeporte/article/view/14405 
Gómez, M., Galeano, C. y Jaramillo, D. (2015). El estado del arte: una metodología de investigación. Revista Colombiana de Ciencias Sociales, 6(2), 423-442. https://doi. org/10.21501/22161201.1469

Hennessey, G. y Rincón, Y. (2018). Liderazgo comunicacional en pequeñas y medianas empresas. En, J. Lechuga, O. Leyva, A. Cazallo, U. Paz, A. Paz, N. Campo, G. Hennessey R. Prieto J. García \& Y. Rincón (Comp.), Liderazgo: tendencias emergentes para inspirar la gestión de personas en las organizaciones (pp. 77-108). Barranquilla: Ediciones UniSimón. Disponible en https://hdl.handle. net/20.500.12442/3841

Hernández, M. y Buitrago, R. (2017). Rol del sinestéta organizacional en el manejo de la comunicación asertiva. Revista Económicas CUC, 38(2), 61-76. http://dx.doi. org/10.17981/econcuc.38.2.2017.05

Hernández, R., Fernández, C. y Baptista, P. (2006). Metodología de la investigación. México, D.F.: McGrawHill.

Martín, S. y Lafuente, V. (2017). Referencias bibliográficas: indicadores para su evaluación en trabajos científicos. Investigación Bibliotecológica, 31(71), 151-180. http://dx.doi.org/10.22201/iibi.0187358xp.2017.71.57814

Montes, F. (septiembre, 2016). La realidad de los gamers y los gamers en la realidad: una aproximación cualitativa. Trabajo presentado en las XV Jornadas de Investigación de la Facultad de Ciencias Sociales, Udelar, Montevideo, Uruguay. Recuperado de http://jornadas.cienciassociales.edu.uy/wp-content/uploads/2016/10/ Eje_CulturaMEdiosyTICS_La-realidad-de-los-gamers_Felipe-Montes.pdf

Parra, M., Durán, S., García, J. y Ramírez, R. (2017a). Gerencia social y motivación laboral en organizaciones de salud pública. En, R. Prieto y A. Cazallo (Comp.), Desarrollo Organizacional y Gestión Humana en Contextos Globalizados (pp. 67-108). Barranquilla. Ediciones UniSimón. Disponible en http://hdl.handle. net/20.500.12442/2602

Parra, M., Durán, S., Romero, C. y Patiño, M. (2017b). Calidad de vida laboral para generar ventaja competitiva en empresas lácteas. En, R. Prieto y A. Cazallo (Comp.), Desarrollo Organizacional y Gestión Humana en Contextos Globalizados. Barranquilla. Ediciones UniSimón (pp. 175-207). Barranquilla: Ediciones Universidad Simón Bolívar. Disponible en http://hdl.handle.net/20.500.12442/2602

Pirela, W., Prieto, R., García, J. y Quijada, G. (2018). Equipos de alto des- empeño como soporte de gestión en industrias camaroneras. En, J. Lechuga, O. Leyva, A. Cazallo, U. Paz, A. Paz, N. Campo, G. Hennessey R. Prieto J. García \& Y. Rincón (Comp.), Liderazgo: tendencias emergentes para inspirar la gestión de personas en las organizaciones (pp. 129-171). Barranquilla: Ediciones UniSimón. Disponible en https://hdl.handle.net/20.500.12442/3841

Prieto, R., Céspedes, J., Palacios, A. y Paz, A. (2017). Competencias de liderazgo en Ediles del Concejo Municipal en las Alcaldías de la Guajira-Colombia. En, R. Prieto y A. Cazallo (Comp.), Desarrollo Organizacional y Gestión Humana en Contextos Globalizados (pp. 11-65). Barranquilla. Ediciones UniSimón. Disponible en http://hdl.handle.net/20.500.12442/2602

Rojas, I. (2011). Elementos para el diseño de técnicas de investigación: una propuesta de definiciones y procedimientos en la investigación científica. Revista Tiempo de Educar, 12(24), 277-297. Disponible en http://hdl.handle.net/20.500.11799/38942 
Roque, R. (2008). Ocio y tiempo libre teorías y enfoques conceptuales en Europa occidental estados unidos de américa. Revista Digital Actívate, (1), 1-45. Disponible en https://www.icoder.go.cr/

Ruz, A. y Lobo, D. (2018). Mercadeo interno: modelo de gestión organizacional en Universidades Privadas de Barranquilla-Colombia. En, J. Lechuga, O. Leyva, A. Cazallo, U. Paz, A. Paz, N. Campo, G. Hennessey R. Prieto J. García \& Y. Rincón (Comp.), Liderazgo: tendencias emergentes para inspirar la gestión de personas en las organizaciones (pp. 109-128). Barranquilla. Ediciones UniSimón. Disponible en https://hdl.handle.net/20.500.12442/3841

Sabariegos, J. (2011). El concepto de trabajo en la economía actual. Desde el pacto social a la precariedad y las distintas reformas laborales. GeoGraphos Revista Digital para Estudiantes de Geografía y Ciencias Sociales, 2(6), 1-24. https://doi. org/10.14198/GEOGRA2011.2.06

Sabino, C. (2006). Los caminos de la ciencia: una introducción al método científico. Buenos Aires: Lumen Humanitas.

Sandoval, N. (2017). Diagnóstico acerca del uso del ocio y el tiempo libre entre los estudiantes de la universidad nacional experimental del Táchira. Pedagogía Social. Revista Interuniversitaria, (30), 169-188. https://doi.org/10.7179/PSRI_2017.30.12

Sandoval, Y. (2009). La industria del ocio, el nuevo consumismo de masas en el Siglo XXI. Topofilia. Revista de Arquitectura, Urbanismo y Ciencias Sociales, 1(3), 1-19. Disponible en http://69.164.202.149/topofilia/index.php/topofilia

Sierra, R. (2010). El método investigativo. Caracas: Editorial Suramérica.

Tamayo y Tamayo, M. (2007). El proceso de investigación científica. México, D.F.: Prentice Hall.

\section{BIODATA}

Ramiro A. Buitrago Acuña es Doctor en Ciencias Gerenciales/ Magister Scientiarum en Gerencia de Recursos Humanos/ Diplomado en Ciencias Forenses y Criminalísticas/ Diplomado en el Procedimiento Penal Venezolano egresado de la Universidad Privada Dr. Rafael Belloso Chacín (URBE). Abogado egresado de La Universidad del Zulia (LUZ). Abogado IV para la Alcaldía Bolivariana del Municipio Miranda (Zulia, Venezuela). Investigador Independiente. http://orcid.org/0000-0002-8563-7662

Josué N. Vera Rodríguez es participante con opción a grado en el Programa Doctoral de Psicología Industrial-Organizacional de la Pontificia Universidad Católica de Puerto Rico (Ponce, Puerto Rico). Maestría en Redacción para los Medios de la Universidad del Sagrado Corazón (San Turse, Puerto Rico). Maestría en Salud Pública General de la Universidad de Puerto Rico (Rio Pierdas, Puerto Rico). Bachillerato en Comunicación Pública de la Universidad de Puerto Rico (Rio Piedras, Puerto Rico). Presidente Ejecutivo para Consultant and Partnership Alliance For Excellence, Inc./ Investigador Independiente. http://orcid.org/0000-0002-0721-5069 